\title{
Docentes vs. estudiantes. Contradicciones en la enseñanza de las ciencias naturales para el desarrollo de competencias científicas ${ }^{*}$
}

\section{Student $v s$. teacher Contradictions in the teaching of science for the development of scientific skills}

\author{
Adriana Castro** y Ruby Ramírez ${ }^{* * *}$
}

Fecha de recepción: 7 de octubre de 2011

Fecha de revisión: 18 de octubre de 2011

Fecha de aprobación: 8 de noviembre de 2011

\section{Resumen}

Se presentan en este trabajo investigativo los resultados de la fase de diagnóstico en la tesis de Maestría en Desarrollo de Competencias Científicas en la Enseñanza

* Artículo con avances investigativos del grupo de investigación denominado Desarrollo institucional integrado, de la Universidad de la Amazonia, y que fue presentado en el marco del I Congreso Internacional de Temas y Problemas en Maestrías y Doctorados en Educación en el 2011.

** Magíster en Ciencias de la Educación, Universidad de la Amazonia. Directora de Formación Pedagógica para el Ingreso a la Carrera Docente. Directora del proyecto Competencias Científicas en la Enseñanza de las Ciencias Naturales. Perteneciente al grupo Ciencias naturales y educación ambiental. Correo electrónico: adrianacas75@yahoo.es

*** Master en Ciencias de la Educación, Universidad del Amazonía, Especialista en Sistemas de Gestión Ambiental, Univ. Externado de Colombia, Ingeniera Agroecológica, coinvestigadora del Grupo: Desarrollo Institucional Integrado. Correo electrónico: rubyrg1@hotmail. com 
de las Ciencias Naturales. Esta fase permitió establecer, de un lado, las contradicciones e incoherencias entre las concepciones de enseñanza de los docentes y sus prácticas de aula; y de otro, la perspectiva de los estudiantes frente a la realidad de las prácticas de enseñanza. Se evidencia que, en el proceso de construcción y reconstrucción del conocimiento, el docente no asume al estudiante como un sujeto activo y protagonista; por el contrario, persisten las prácticas tradicionales de enseñanza y aprendizaje.

Palabras clave: enseñanza de las ciencias naturales, aprendizaje, competencias científicas, concepciones.

\begin{abstract}
This research work presents the results of the diagnostic phase in the master's thesis Development of scientific expertise in the teaching of natural sciences. This phase allowed us to establish on the one hand, the contradictions and inconsistencies between conceptions of teaching from teachers and their classroom practices, and on the other, the perspective of the students on the reality of teaching practices. It is evident that, in the process of construction and reconstruction of knowledge, the teacher does not assume the student as an active subject and protagonist, but on the centrary traditional practices of teaching and learning persist.
\end{abstract}

Keywords: Natural science teaching, learning, scientific skills, conceptions.

\section{Introducción}

La globalización y la revolución científica han aumentado la importancia de la enseñanza y aprendizaje de las ciencias naturales, así como el valor de las actitudes activas y conscientes para la apropiación de conocimientos. Los nuevos retos de un mundo cambiante en todas sus dimensiones, especialmente en el aporte científico y la relación del hombre con el entorno ambiental, han llevado a resaltar la importancia de la investigación en didáctica de las ciencias naturales para la generación de conocimiento científico que permita mejorar la calidad de su enseñanza y aprendizaje, especialmente, en la dirección de contribuir al desarrollo de competencias científicas en el estudiante. Perspectiva que representa un enorme reto para el maestro y el país, cuando "hoy América Latina apenas genera el $2,5 \%$ del conocimiento científico de frontera, y tan solo diez países altamente desarrollados producen el $94 \%$ del saber y la tecnología de punta (Bravo, 2003, p. 24). Si esta realidad no se instala en el centro de la problemática de la enseñanza y el aprendizaje de las ciencias en todos los niveles educativos, el 
propósito nacional sobre desarrollo de competencias científicas carecería de solidez. Por ello, asumir este reto es una gran responsabilidad, especialmente por las implicaciones didácticas y curriculares para docentes, estudiantes e instituciones educativas.

En Colombia, la Ley 115 de 1994 establece la formación científica básica como fin de la educación (artículos 5, 7, 9 y 13). Para alcanzar dichos fines las competencias son adoptadas por organismos nacionales como el Ministerio de Educación Nacional (MEN) a través de la expedición de los lineamientos curriculares y estándares de competencias. Parece, entonces, en el sentir internacional y nacional, que las competencias permean los sistemas educativos, para generar el desarrollo de una cultura científica en los niños y jóvenes.

No obstante, en la educación básica secundaria son reducidos los esfuerzos para el fomento de procesos investigativos que permitan desarrollar capacidades como la curiosidad, el deseo de conocer, plantearse preguntas, observar, criticar, reflexionar y solucionar problemas. Esto ha dificultado el desarrollo de competencias científicas en el estudiante, que le permitan una aproximación cultural a la ciencia y la tecnología desde la escuela. Asimismo, existen debilidades en los estudiantes frente al desarrollo de capacidades como: re-construir teorías, formular hipótesis, diseñar experimentos, argumentar, imaginar, ser creativos y construir alternativas de solución a problemas del entorno, objetivos reiterados en los lineamientos curriculares y estándares de competencias. De acuerdo a estos planteamientos se hace necesario responder a nuestra pregunta de investigación: ¿cómo desarrollar en los estudiantes competencias científicas mediante la enseñanza de ciencias naturales en la educación básica secundaria?

En esta perspectiva, la metodología asumida en la fase diagnóstica es de corte interpretativadescriptiva, mediante la integración de métodos cualitativos y cuantitativos que permitan la percepción directa del objeto de investigación y del problema. El objetivo es establecer las concepciones de enseñanza de los docentes y sus prácticas de aula frente a la propuesta nacional oficial e institucional, así como la perspectiva de los estudiantes frente a la realidad de las prácticas de enseñanza y sus implicaciones para el desarrollo de competencias científicas, desde los enfoques didácticos (resolución de problemas), los recursos de aprendizaje, espacios de aprendizaje y las prácticas evaluativas.

\section{Referentes teórico-conceptuales}

Los referentes teóricos que orientan esta ponencia se inscriben en dos categorías de análisis: naturaleza de las ciencias naturales contenidas en la situación de enseñanza y la enseñanza de las ciencias naturales y el desarrollo de competencias científicas.

\section{Naturaleza de las ciencias naturales contenidas en la situación de enseñanza}

La reflexión filosófica de la ciencia se aborda inicialmente desde los planteamientos de Husserl (citado en MEN, 1998), con su concepto 
del mundo de la vida, en donde afirma que la misma es:

Todo lo que compartimos, científicos y no científicos (...). El mundo de la vida y el mundo de las teorías científicas se suelen contraponer, el mundo de la vida es un mundo de perspectivas, cada quien lo ve desde su propio punto de vista, en el mundo de la ciencia los científicos intentan llegar a acuerdos intersubjetivos y para ello se debe llegar a consensos, en otras palabras abandonar sus propias perspectivas situándose en diferentes puntos de vista que permitan llegar a una síntesis objetiva, o mejor aún, intersubjetivas (p. 6).

Desde esta visión filosófica, enseñar ciencias implica relacionar al estudiante con la oportunidad de establecer un diálogo racional entre su propia perspectiva y las demás, con el fin de explicarse el mundo de manera más científica en forma progresiva.

Hernández (2005) hace su análisis desde el punto de vista epistemológico poniendo en consideración la caducidad de la lógica deductiva y proclama el triunfo del constructivismo. Ante esta perspectiva, la epistemología constructiva pone en crisis la fe absoluta en los principios de la ciencia y recuerda que estos se construyeron de manera inductiva, a partir del modelo de la experimentación demostrativa y que la escuela tiene el deber de promover en los estudiantes habilidades para plantear y validar sus propias hipótesis y diseñar estrategias de acercamiento a la realidad. Por ello, es importante reflexionar sobre la naturaleza de la enseñanza de las ciencias naturales para poder dar sentido y relevancia didáctica al desarrollo de las competencias científicas en los estudiantes. Según Mora (1997), se debe tener cuidado con un enfoque de las competencias basado en un paradigma tecno-científico que olvida el elemento humanístico de las mismas:

La competencia desenfrenada por la producción de bienes tecnológicos que satisfagan el afán por el bienestar y seguridad material, ha influido poderosamente para que se haya convertido la ciencia en una moderna religión portadora de verdades universales, métodos irrefutables y saberes no contaminables por la subjetividad (p. 137).

Por otro lado, es importante considerar que la ciencia es siempre inacabada. Día a día se construyen nuevas teorías y nacen nuevos conceptos que responden a nuevas realidades del mundo. No se debe concebir la verdad como absoluta y perenne, este es uno de los problemas más comunes en la enseñanza de las ciencias naturales. Los estudiantes generalmente creen que la realidad la encontrarán en los libros y pocas veces son conscientes de que los diversos modelos y teorías que estudian, pueden cambiar con el tiempo y ser superados por otros. Es esencial para el profesor de ciencias asumir la provisionalidad y falibilidad del conocimiento.

Desarrollar competencias científicas a temprana edad en el estudiante le permite apropiar la cultura científica y hacer de su aprendizaje un proceso significativo, esto es confirmado por Mora (1997), al afirmar que: 
[...] durante el proceso escolar el desarrollo en el niño de una imagen correcta sobre la naturaleza de las ciencias y los procesos de producción del conocimiento científico, influye de manera significativa, no solo en la cultura general, sino particularmente para despertar interés, motivación en la juventud que va a seguir estudios en las diferentes carreras científicas y tecnológicas (p. 139).

No se puede continuar cayendo en el error de considerar a la ciencia como acumuladora de conocimientos verdaderos, porque podríamos estar estancándola y limitando en los estudiantes procesos de criticidad, curiosidad, creatividad y confianza en sí mismos.

Se deben propiciar las condiciones para que la enseñanza de las ciencias naturales no se limite a memorizar algunos de los resultados logrados en un determinado momento de la historia de la ciencia, hay que propiciar espacios para que se generen preguntas y respuestas que ejerciten la controversia, la experimentación y la crítica para permitir conocer el mundo de manera científica. El desarrollo de competencias científicas invita a la exploración de hechos y fenómenos naturales, al análisis de problemas. La observación, la utilización de diferentes métodos de análisis y recolección de información, son algunos de los procesos que se deben iniciar para comprender la ciencia y poder de esta manera transformarla y reconstruir conocimientos que den sentido a la enseñanza de las ciencias naturales.

Establecer el contraste entre el conocimiento común, científico y tecnológico permite diferenciar sus intereses y las formas como se construyen. Para el Ministerio de Educación Nacional (MEN, 1998), existen diversos tipos de conocimientos: el común u ordinario que es el que construye el hombre como actor en el mundo de la vida; existe también el conocimiento científico y el tecnológico. Las propiedades que distinguen el conocimiento común del científico y tecnológico son: el conocimiento común no se preocupa por la construcción de teorías que vinculen hechos, el hecho por sí solo es satisfactorio; por el contrario, para el científico y tecnólogo lo más importante es la intención teórica, que entre más general sea la teoría más relevancia tiene para la comunidad científica dedicada a esa área del conocimiento. La segunda diferencia es que el conocimiento científico y tecnológico son productos de un proceso de producción social que es sometido a un exigente proceso de legitimación por el sistema que lo produce para ser aceptado universalmente por los miembros del sistema social en cuestión; contrario a esto, el conocimiento común no necesita de este proceso para ser legitimado, sino que es un acontecimiento individual, depende de los individuos que lo aceptan como válido.

Soto (2002) analiza la relación entre el conocimiento cotidiano y el conocimiento científico escolar en la esfera de la educación en ciencias. El autor analiza las diferentes teorías constructivistas y no constructivistas desde distintas concepciones:

- En un primer plano ubica la concepción de que los conocimientos cotidiano y científico no son sino formas distintas de un solo tipo de conocimiento, es decir, hay continuidad 
entre las formas de construir conocimiento individual, y las formas de construir conocimiento científico escolar.

- La segunda concepción tiene que ver con una supuesta incompatibilidad epistemológica entre el llamado "conocimiento cotidiano" y las estructuras del pensamiento científico. Este planteamiento central implica la existencia de un salto cualitativo que no permite ubicar estos dos conocimientos en un mismo nivel.

- La tercera concepción plantea que los conocimientos cotidiano y científico son formas de conocimiento diferentes, contextualmente distintas en la manera en que se piensa, se razona, se organiza cognitivamente el mundo, y que por consiguiente persiguen metas y fines distintos (p. 14).

Porlán (1995) hace sus aportes a esta clasificación aclarando la complejidad del conocimiento cotidiano. Su enriquecimiento y maduración hacia formas relativas de autonomía, es la estrategia adecuada para una regeneración democrática de la ciencia y para la incardinación dialéctica en los procesos de reflexión crítica del pensamiento humano. Para este investigador, los rasgos comunes que poseen los diversos tipos de conocimiento son: primero, todo conocimiento implica una representación mental (lingüística, pictórica, kinestésica, auditiva, etc.) de aquello que se conoce; un segundo rasgo es que todo conocimiento se hace posible dentro de un contexto social y el tercero es que el conocimiento tiene un valor adaptativo al mundo físico o socio-cultural e individual. De esta manera, el autor manifiesta que "desde un punto de vista educativo, se trabaja desde y para el conocimiento que tienen, generan y construyen los alumnos" (p. 104). De lo anterior es posible inferir que el conocimiento escolar, si bien no puede considerarse conocimiento científico, sí tiene como marco de referencia el conocimiento científico que ha sido previamente construido y asumido como tal por la humanidad. Por esto, el docente de ciencias naturales ha de instalar su saber disciplinar y su saber pedagógico, didáctico y curricular en el marco de las teorías más avanzadas de la disciplina científica y de su enseñabilidad.

\section{Enseñanza de las ciencias naturales y desarrollo de competencias científicas}

El concepto de competencia en el contexto educativo y en las ciencias naturales es un término que ha alcanzando importancia en las sociedades del conocimiento. Maldonado (2002) explica que su origen se encuentra en el mundo laboral y profesional tanto para Europa como para América Latina hacia los años sesenta, propuesta inspirada en la producción empresarial en donde se vincula la formación para el trabajo. Posteriormente, en los años setenta, el alemán Gerhard Bunk introduce este término en el marco del mundo educativo y el mundo laboral definiéndolos desde una perspectiva de capacidades entendidas como conocimientos, destrezas y aptitudes para ejercer una profesión.

Existen variadas concepciones de competencia que circulan en el contexto escolar. Escobedo (2001) afirma que "desde la perspectiva de la educación para ser competente en un 
determinado campo, es necesario conocer y comprender, poder cooperar armónicamente con los demás, ser sensible a los problemas del campo y sentir gusto en trabajar para tratar de resolverlos" (p. 47). Por otro lado, y desde la didáctica de las matemáticas, Bruno D'Amore (2008) afirma "la competencia como un concepto complejo y dinámico: complejo porque se trata del conjunto de dos componentes: el uso (exógeno) y el dominio (endógeno), incluso de elaboración cognitiva, interpretativa y creativa, de conocimientos que relacionan contenidos diferentes" (p. 12). Para el autor, la competencia requiere factores metacognitivos: la aceptación del estímulo para usarlos, el deseo de hacerlo, el deseo de completar los conocimientos que se revelan a la prueba de los hechos insuficientes, y por tanto el deseo mismo de aumentar la propia competencia. La competencia no es sólo el uso y el dominio de dichos conocimientos, sino también un conjunto de actitudes que muestran la disponibilidad afectivamente positiva de desear hacer uso. ¿Qué sería de una competencia sin el deseo, sin la voluntad y sin el gusto de hacer uso de ella? (p. 21).

Sin embargo, el concepto de competencia tiende a ser conceptualizado de una manera instrumental y eficientista en la educación, asumiendo exclusivamente competencias en lo cognitivo, las habilidades y destrezas del dominio que se trata. Es importante reconocer que la propuesta nacional en la enseñanza de las ciencias naturales desarrolla esta concepción, especialmente la de capacidades y saberes para relacionar las competencias científicas; pero, aunque prima el énfasis en lo cognitivo, no desconoce el saber ser (com- ponente volitivo), al añadir el componente de compromisos sociales que debe desarrollar el estudiante en el aula.

Una vez conceptualizado el término competencia, se requiere identificar su ubicación desde la enseñanza de las ciencias naturales. Escobedo $(2001$, p. 47) afirma que una persona es competente para ser productiva en las ciencias naturales cuando ha logrado:

- Desarrollar el pensamiento científico implica: comprender los procesos de lo real y construir teorías científicas acerca del mundo natural que permitan predecir y controlar los fenómenos naturales; manejar el lenguaje de la ciencia de manera oral y escrita, lo que implica exponer, argumentar en forma lógica, producir informes descriptivos o explicativos y conocer la forma como uno conoce $(\ldots)$

- Desarrollar la capacidad de trabajar en equipo. Implica escuchar y entender los argumentos de los demás, apreciar y resaltar el trabajo de los demás haciendo énfasis al respeto de las personas, hacer sugerencias valiosas y productivas para el conocimiento científico $(\ldots)$

- Desarrollar el interés por el conocimiento científico. Implica desarrollar la sensibilidad para detectar problemas científicos, emprender con entusiasmo y placer la tarea de resolver problemas planteados (...) (p. 48).

Se resalta en este autor una concepción que no se agota en lo cognitivo de la competencia y reconoce su complejidad e integralidad, al igual 
que lo plantean Hernández (2005), Quintanilla (2005), Cañas, Díaz y Nieda (2007) y D'Amore (2008) al resaltar en las competencias los componentes cognitivos, procedimentales y actitudinales indispensables en la enseñanza y que deben ser asumidos por y desde diferentes ámbitos de la vida en los que se movilizan de manera interrelacionada.

Muchos de nosotros somos el resultado de la enseñanza de las ciencias naturales sin asistir nunca o casi nunca al laboratorio. Somos resultado del aprendizaje memorístico de los temas contenidos en los textos, los cuales eran verdades absolutas e incuestionables; casi nunca relacionábamos los temas con la vida cotidiana. Aunque esta estrategia prevalezca aún en algunas instituciones para la enseñanza y aprendizaje de las ciencias naturales, está siendo sustituida por otras formas de enseñanza centradas en el contacto directo con los fenómenos naturales y en la participación explícita y consciente de los alumnos en la producción de sus conocimientos.

Estos cambios en la enseñanza de la ciencias naturales, responden a las necesidades actuales de nuestra sociedad, en donde las personas deben poseer ciertas competencias científicas y además, poder desarrollar habilidades lógicas de pensamiento. Así, estar informados y capacitados permite apropiarse de los nuevos contenidos de los diferentes campos conceptuales y comprender mejor la realidad.

El ciudadano de hoy requiere una formación básica en ciencias si aspira a comprender su entorno y a participar en las decisiones sociales. La enseñanza de las ciencias es parte esencial de la formación de ese ciudadano. Se trata de desarrollar en la escuela las competencias necesarias para la formación de un modo de relación con las ciencias (y con el mundo a través de las ciencias) coherentes con una idea de ciudadano en el mundo de hoy (Hernández, 2005, p. 2).

La realidad cambia todos los días, y el hombre es el responsable de transformar esa realidad y modificarla a sus necesidades a partir de la toma de decisiones que surgen del análisis de los problemas que lo rodean. Ser críticos y capaces de interpretar dichos problemas, investigar y adoptar tecnología, son algunos de los aspectos por los cuales se torna importante la enseñanza y formación en las ciencias naturales para que haya apropiación del conocimiento científico necesario para realizar este propósito.

Cuando se enseña ciencias naturales, se requiere retomar y seleccionar determinados contenidos científicos para hacerlos enseñables. Zambrano (2005) afirma que las categorías del discurso del saber son: el saber-científico, que se refiere a las construcciones científicas, que da cuenta de cómo un campo de la ciencia organiza y elabora sus teorías; el saber objeto de enseñanza, que es el cuerpo teórico de la disciplina científica, obedece a la lógica de la ciencia, es el saber sabio de Chevallard; y el saber enseñado, con el que se identifican las ciencias naturales en el acto práctico de la enseñanza, "es decir, es aquel saber que los sujetos aprehenden aunque parezca mediado entre el saber actitudinal del docente y el saber práctico del alumno" (p. 50), es la forma de hacer visibles los conceptos científicos en 
el lenguaje común en el que se desenvuelven los estudiantes, obedece a la lógica de la asignatura o área de enseñanza, es producto de la mediación didáctica y debe contribuir al desarrollo de competencias científicas.

En la enseñanza de las ciencias naturales, el docente es la persona que posibilita la transformación intelectual, afectiva y moral de los estudiantes. Debe ser consciente de que con su trabajo está contribuyendo al desarrollo científico y tecnológico del país y por consiguiente a la calidad de vida. El docente debe ser investigador pedagógico, conocer el medio donde se realiza la actividad de formación integral para enfrentar con éxito la vida contemporánea fuertemente influenciada por el desarrollo científico y tecnológico y la problemática sociocultural y ambiental.

Una estrategia para el desarrollo en los estudiantes del deseo de aprender e indagar permanentemente, son las actividades experimentales, que junto con la resolución de problemas, permiten el desarrollo de competencias científicas básicas. Las prácticas de laboratorio y de campo permiten acercar al estudiante con la realidad y comprender de esta manera fenómenos naturales. EI MEN (1998) explica que "las actividades experimentales o prácticas de laboratorio tienen el papel de confirmar o falsear las hipótesis que el científico ha construido sobre la base de sus idealizaciones del mundo de la vida" (p. 91). El estudiante debe ir al laboratorio conociendo el tema, saber qué es lo que va a observar, conocer los procedimientos y el proceso de toma de datos. Debe ser el que formula las hipótesis luego de un proceso de reflexión y discusión del tema. Así mismo, el docente debe motivar y brindar los espacios para que los estudiantes diseñen sus propios experimentos y desarrollen capacidades creativas, argumentativas e interpretativas.

Estas capacidades se desarrollan a partir de procesos de resolución de problemas, base de la investigación en el aula. Por tal motivo, la planificación de los procesos investigativos debe vincular actividades en donde el tema seleccionado por el docente, sea redescubierto por los estudiantes en búsqueda de los aspectos más interesantes para ellos, plantear problemas significativos, identificar concepciones de los alumnos frente al tema, contrastar información para abordar obstáculos en el aprendizaje y aplicar los conocimientos a situaciones prácticas.

El planteamiento y tratamiento de problemas ha sido una estrategia empleada en la enseñanza de las ciencias naturales para el desarrollo de competencias científicas y sugeridas por el MEN como estrategia para desarrollar actitudes científicas en los estudiantes:

El desarrollo de actitudes hacia las ciencias, puede ser generado por procesos de intervención pedagógica que implique una enseñanza adecuada de las ciencias, que estén basados en un modelo didáctico que presente estrategias sistemáticas que modifiquen o transformen estas actitudes, un modelo que esté fundamentado en la resolución de situaciones problemáticas que, además de generar actitudes adecuadas hacia las ciencias, provoque desarrollos en 
la independencia cognoscitiva, la capacidad creativa y la construcción de conocimientos en los estudiantes (García, 2003, p. 39).

Conviene observar que los docentes orientan su actividad en el aula según las concepciones que posean de la naturaleza de las ciencias; sin embargo, las concepciones contemporáneas promovidas en la propuesta oficial no permean la práctica docente. Para Florez, Gallegos, Bonilla, López y García (2007), en docentes de biología de básica secundaria la tendencia en la concepción de ciencia se encuentra en la corriente filosófica del positivismo, porque buscan el conocimiento objetivo y acumulativo. Por tanto, esta concepción no se acerca a las tendencias actuales de la construcción del conocimiento científico; por el contrario, se acerca a una concepción tradicional centrada en la transmisión de información y el aprendizaje memorístico y acumulativo.

\section{Aspectos metodológicos}

El diseño de esta fase requirió de un enfoque de complementariedad de métodos (polifonía de métodos cuantitativos y cualitativos) para abordar de forma integral el fenómeno educativo relacionado con la investigación, y de esta manera determinar la evolución y estado actual del problema, en el caso particular de las instituciones educativas que hacen parte de la muestra poblacional.

En cada institución educativa se aplicaron dos instrumentos a docentes y dos a estudiantes para la recolección de la información, así:
- Encuesta y entrevista a docentes del área de ciencias naturales de la básica secundaria.

- Encuesta y grupo focal a estudiantes del grado noveno.

- Se tuvieron en cuenta los siguientes métodos:

- Métodos de interrogación: a partir del diseño y aplicación de encuestas, entrevista y grupo focal.

- Métodos de nivel matemático estadístico: se usaron software específicos para el análisis de encuestas como SPSS (docentes) y encuestas AS (estudiantes), para establecer la dimensión cuantitativa del problema, tabulación, análisis y sistematización de los datos obtenidos.

Para la aplicación de instrumentos en las instituciones educativas se realizó la preparación de las condiciones, socializando la naturaleza y objetivo de la investigación, aclarando las inquietudes de docentes y estudiantes que hicieron parte del diagnóstico y estableciendo un ambiente adecuado para la implementación de los instrumentos.

\section{Encuesta a estudiantes}

Se aplicaron cuarenta y ocho encuestas a estudiantes del grado noveno de básica secundaria. El instrumento recolectó información de los siguientes elementos: razones por las cuales existe interés hacia el aprendizaje de las ciencias naturales, actividades que realiza el docente y el estudiante en las clases de ciencias naturales, formas de evaluación. Los 
datos obtenidos se sistematizaron en el software encuesta AS. Se construyeron los códigos tipo Lista Check Box, valores excluyentes, texto libre y número para el procesamiento de la información cuantitativa, elaborando el informe de frecuencias con el que se establecieron los hallazgos de mayor representatividad e importancia para la investigación. La información cualitativa se analizó desde el procesador de texto, manejando códigos por colores para cada una de las categorías que emergieron de los datos.

\section{Encuesta a docentes}

Se aplicaron cuatro encuestas. La aplicación de las encuestas se hizo de manera individual, tomando cada docente el tiempo necesario para su diligenciamiento. El instrumento recolectó información de los siguientes elementos: relación de la práctica docente con las políticas educativas del MEN, enseñanza de las ciencias naturales y el desarrollo de competencias científicas, plan de estudios institucional e incorporación de competencias, actividades que se desarrollan en las clases de ciencias, uso del laboratorio, competencias que se desarrollan en las clases de ciencias naturales, actividades para fomentar la investigación y recursos pedagógicos y didácticos empleados en las clases. El procesamiento y sistematización de la información se efectuó en el software SPSS, previa construcción de códigos numéricos, en cadena y medida nominal.

\section{Entrevista a docentes}

Este instrumento se construyó para profundizar en aspectos particulares surgidos de la encuesta que, por su importancia, contribuyen a ampliar el análisis cualitativo de la información que los docentes podían aportar para establecer el estado actual del problema. Por esta razón, se indagó sobre la concepción de ciencias y propósito de la enseñanza, desarrollo de la clase de ciencias naturales, competencias que promueve desarrollar en los estudiantes, estrategias didácticas que emplea para desarrollar las clases, recursos didácticos utilizados, concepción de evaluación del aprendizaje y cómo evalúa a los estudiantes. El análisis de los resultados se efectuó mediante el uso de descriptores (palabras) para las categorías de análisis y (colores) para diferenciar los docentes de cada institución.

\section{Grupos focales a estudiantes}

La aplicación de esta técnica de investigación cualitativa surge de la necesidad de ampliar aspectos que requerían aclararse luego de procesar estadísticamente las encuestas. Logramos determinar que algunos términos usados en las encuestas a estudiantes generaron dualidades en su concepción por parte de los encuestados, lo que arrojó datos dudosos que necesitaban de mayor indagación. De la muestra empleada para la encuestas se seleccionaron seis estudiantes en cada institución educativa, teniendo en cuenta criterios establecidos (edad, sexos, rendimiento académico, facilidad de expresión, perspectiva crítica y analítica). La información se procesó en forma digital. El análisis se 
realizó de igual manera que las entrevistas mediante el uso de descriptores (palabras) para las categorías de análisis y (colores) para diferenciar los grupos de estudiantes de cada institución.

\section{Triangulación}

Inicialmente, se elaboraron informes descriptivos de los resultados de las encuestas a docentes y estudiantes (datos cuantitativos) y se procesaron las entrevistas y grupos focales con descriptores y colores (datos cualitativos). La organización de los datos permitió establecer unas categorías y subcategorías de análisis; su tratamiento se efectuó en una matriz comparativa que se nutrió con cada uno de los aportes de las fuentes de información (docentes-estudiantes) y permitió contrastar de esta manera las concepciones, orientaciones didácticas, recursos, ambientes de aprendizaje y tipos de evaluación desde la mirada de cada uno de los autores del proceso pedagógico.

\section{Resultados alcanzados y esperados}

Los resultados de la fase diagnóstica se presentan teniendo en cuenta dos elementos fundamentales: el primero relacionado con el análisis de la propuesta nacional oficial y la institucional para la enseñanza de las ciencias naturales; y el segundo, el análisis de las contradicciones e incoherencias identificadas a partir del estudio de las perspectivas de docentes y estudiantes.
La enseñanza de las ciencias naturales en Colombia está orientada por los lineamientos curriculares (MEN, 1988) y Estándares de competencias (MEN, 2004), los cuales dan las pautas a los docentes para orientar el proceso educativo y establecer criterios de calidad de la enseñanza de las ciencias.

En estos documentos oficiales se encontró que la concepción de competencia se relaciona con términos como capacidades, habilidades, aptitudes, saber conocer, saber hacer y saber ser. La concepción de competencia científica se orienta a la aproximación de los estudiantes al conocimiento científico privilegiando el razonamiento lógico, la argumentación escrita y oral, la experimentación, el uso de la información científica y la apropiación del lenguaje duro de la ciencia y la tecnología. Las competencias científicas van desde la construcción y manejo de conocimientos hasta el desarrollo de capacidades investigativas que se entienden como la ruta o camino a seguir en búsqueda del desarrollo del pensamiento científico. Y la adopción de posturas éticas en la relación del hombre con la ciencia, tecnología y sociedad. Es conveniente resaltar que esta investigación realizó los acercamientos de las competencias desde conceptos relacionados con habilidades y capacidades según Hernández (2005), Maldonado (2002) y Tobón (2006).

La estructura de la propuesta oficial visibiliza aspectos centrados en el desarrollo cognitivo de los estudiantes cuando relaciona ejes fundamentales que describen acciones concretas de pensamiento y acción, y aspectos de un componente social y cultural cuando proponen 
la formación de una conciencia ética sobre el papel de las ciencias naturales en relación con el ambiente, la calidad de vida y el compromiso con el trabajo científico y la sociedad. Estos componentes se asumen en tres procesos formativos que se acercan a las dimensiones del saber asumidas en la investigación como el saber-conocer, hacer y ser referenciados desde las posturas de Hernández (2005), Quintanilla (2005) y Tobón (2006).

Se evidencian en la propuesta nacional oficial acercamientos al paradigma constructivista de la educación cuando se identifica que la estructura curricular propuesta se organiza teniendo en cuenta "niveles de complejidad de los procesos de pensamiento y acción" (MEN, 1998, p. 120), sustentada en que "el desarrollo del pensamiento en niños y niñas avanza poco a poco hacia formas más complejas, la formación en ciencias debe respetar este desarrollo y a la vez jalonarlo" (MEN, 2004, p. 109). De esta manera, presenta una influencia piagetiana confirmada al plantear el rol del maestro como "un docente que no se limite a la transmisión de conocimientos o demostración de experiencias, sino que oriente el proceso de investigación de sus estudiantes como un acompañante" (p. 111).

En cuanto a los documentos institucionales analizados como el Proyecto Educativo Institucional (PEI) y los planes de estudio de cada una de las instituciones estudiadas, se encontró que en el PEI el término competencia aparece en diversos componentes pero no se visibiliza una apropiación del concepto que permita su coherencia en todo el documento. Los planes de estudio se acercan a la propuesta oficial nacional desde los estándares de competencias porque se identifican temáticas y competencias básicas y específicas abordadas desde el saber conocer, saber hacer y saber ser.

A nivel institucional, escasamente se encuentran referentes teóricos y metodológicos que permitan orientar la enseñanza y aprendizaje de las ciencias naturales. Existen algunos acercamientos al constructivismo, cuando asumen el rol protagónico del estudiante en su formación, participando activamente en la construcción del conocimiento a partir de la problematización de las relaciones que establece consigo, con los demás, con el conocimiento y con el entorno. Así mismo, ambas propuestas resaltan el valor de la construcción del conocimiento científico desde una visión biocéntrica, porque pretenden restablecer el valor primordial de la vida, la cultura de lo vivo, la complejidad de las relaciones entre el ser humano y su ambiente.

Sin embargo, existen rupturas filosóficas, epistemológicas y metodológicas entre la propuesta oficial nacional y la institucional para la enseñanza de las ciencias naturales, las cuales tienen implicaciones en el aspecto didáctico, es decir, lo que realmente hace el docente en el aula de clase. Al indagar sobre la concepción de competencia que los docentes tienen mediante el análisis de sus respuesta sobre el propósito de la enseñanza de las ciencias, se hace explícito el interés por desarrollarlas: "la competencia que yo busco desarrollar en el alumno es la competencia científica". De igual manera, en los resultados de la encuestas a los docentes se estableció que el $80 \%$ sí creen que 
la enseñanza de las ciencias naturales estimula el desarrollo de competencias científicas.

Las concepciones de los docentes sobre las competencias, direccionan el término a la formación integral del individuo. De tal manera que expresiones como: "aprenda a indagar, aprenda a conocer su medio", "proyección hacia la conservación del medio ambiente, recursos naturales en general", "aprenda el estudiante a conservar el medio ambiente para que sea un investigador, un indagador de su entorno", "todos los muchachos en general eh tomen conciencia", "desde el punto de vista experimental, observación, eh la parte de la explicación, y también pues conociendo las necesidades del entorno, y también mirando la posibilidad que tenga la aplicación de ese entorno hacia la realidad del medio", "el alumno se vaya formando una idea con las concepciones que él tiene del mundo de las cosas", visibiliza que la concepción de competencia de los docentes integra los conocimientos, procedimientos y actitudes en el proceso de formación acercándose a la propuesta nacional oficial.

Sin embargo, el discurso docente sobre competencia es aún incipiente, lo que dificulta de forma significativa su empleo en la enseñanza de las ciencias naturales. Según Tobón (2006), estos vacíos se relacionan con la concepción reduccionista, el pensamiento acrítico y descontextualizado y la inconsistencia y falta de claridad en la estructura conceptual del término competencias.

Con respecto a las orientaciones didácticas para el desarrollo de las competencias científicas, la propuesta nacional oficial se aproxima al paradigma constructivista y da prioridad al planteamiento de preguntas y la solución de problemas para la construcción del conocimiento a partir de la reflexión; para esto, resaltan la importancia del papel del laboratorio en el diseño y realización de experimentos que permitirán a partir de una pregunta sentir la curiosidad típica del científico y dar respuesta a ese interrogante que le exige poner en funcionamiento su capacidad de razonar (MEN, 1998, p. 91). Las perspectivas docentes sobre la enseñanza de las ciencias naturales priorizan igualmente acciones tendientes a la apropiación de conceptos y procesos experimentales que permitan articular la teoría con la práctica. De este modo, resalta la importancia del laboratorio y la investigación.

Se evidencia que las concepciones del docente de ciencias naturales sobre la enseñanza por competencias, corresponden con la propuesta nacional oficial. Sin embargo, al indagar sobre las percepciones de los estudiantes frente a la práctica docente en el aula se identifican contradicciones que analizaremos a continuación.

Las orientaciones constructivistas asumidas en los modelos pedagógicos y las concepciones docentes riñen con la realidad. Se evidencia que el docente en la construcción de conocimiento no asume al estudiante como protagonista de este proceso y no parte del aprender haciendo. Así lo muestran los resultados de las encuestas a estudiantes en donde las salidas de campo, prácticas de laboratorio y procesos de investigación superan porcentajes del $70 \%$ en las opciones "nunca" $\mathrm{y}$ "algunas veces". 
Entre las actividades fundamentales a disposición del profesor para que los estudiantes aprendan ciencias naturales, se encuentra la experimentación, y son ellos quienes resaltan la importancia del laboratorio para el desarrollo de las competencias científicas. Sin embargo, para los estudiantes estas clases carecen de procesos experimentales y espacios que permitan llevar la teoría a la práctica. Un $68 \%$ de los estudiantes afirman que "nunca" van al laboratorio o realizan experimentos. Para muchos de ellos el laboratorio no es más que otra aula de clase en condiciones más adecuadas y cómodas para recibir la teoría y no representa el espacio que permita la práctica para refutar o comprobar las hipótesis a preguntas planteadas sobre su relación con el entorno.

El estudiante relaciona una clase de ciencias naturales con términos como laboratorios y experimentos, actividades básicas para poner a prueba las hipótesis de los estudiantes frente a sus perspectivas del mundo y poder comprenderlas. En este caso, la tendencia en los docentes va dirigida a la poca experimentación, limitando el aprendizaje sólo a la teoría. Los docentes que escasamente realizan experiencias en el laboratorio se ajustan a los resultados de la teoría, es decir, esperan que suceda lo que dice la teoría que debe suceder, "la experimentación no debe ser ilustrativa, debe convertirse en un instrumento para construir conocimiento válido y convincente a partir de procesos de argumentación" (Escobedo, 2001, p. 69). Es decir, la experimentación debe responder a las expectativas de grupos de estudiantes que defienden teorías opuestas desde sus perspectivas. Cabe resaltar que la experimentación no debe limitarse a diseños controlados por el docente, este debe proporcionar las condiciones para que el experimento se adapte a las condiciones y vida cotidiana del estudiante. Asimismo, estos procesos no necesariamente se pueden realizar en un aula llamada laboratorio; por el contrario, se pueden desarrollar desde otros espacios fuera de la jornada escolar.

Esto también evidencia que el paradigma constructivista, identificado en las propuestas pedagógicas de las instituciones, no es coherente con las actividades de aula; la experimentación es una alternativa didáctica para que el alumno ponga a prueba sus propias hipótesis sobre su entendimiento del entorno. Para Escobedo (2001) "la experimentación coherente con la epistemología piagetiana no puede ser tan diseñada y controlada, ni tiene que desarrollarse en el medio escolar" (p. 68). Consideramos que una de las limitantes de los escasos procesos de experimentación es considerar precisamente la relación directa de esta con el aula de laboratorio por parte de estudiantes y docentes y el desconocimiento de las diferentes formas y espacios de experimentación.

Con respecto a la práctica docente se estableció que el $100 \%$ de los docentes tienen una tendencia al desarrollo de talleres y el trabajo en grupo. Esto es confirmado por los estudiantes en un $80 \%$. Sin embargo, los trabajos constan específicamente en el desarrollo de talleres de los libros guía.

No cabe duda de que el desarrollo de talleres desde el punto de vista pedagógico permite integrar la teoría con la práctica si se tiene en 
cuenta su significación pedagógica. En este sentido, el término taller es relacionado por parte de docentes y estudiantes con actividades contenidas en los libros de texto guía del docente. Según Ander (1991), "el taller es una forma de enseñar y sobre todo de aprender, mediante la realización de algo, que se lleva a cabo conjuntamente, es un aprender haciendo en grupo" (p. 10). No obstante, según los estudiantes el trabajo en grupo escasamente responde a una participación activa y cooperativa. El trabajo en grupo no implica entender y escuchar los argumentos de los demás desde sus perspectivas, resaltar los aspectos positivos del trabajo de los compañeros, hacer sugerencias pertinentes, valiosas y productivas necesarias para el conocimiento científico; por el contrario, es la opción de trabajo por la falta de los recursos didácticos.

El planteamiento de problemas según la propuesta oficial es la mejor manera para iniciar un tema científico, según el $100 \%$ de los docentes "casi siempre" realizan actividades de planteamiento y solución de problemas. Sin embargo, esta alternativa didáctica se limita a la formulación y respuesta a preguntas para indagar o aclarar dudas de un tema específico. Así mismo, según los estudiantes los problemas corresponden a los establecidos en los textos guía del docente. Esta perspectiva sobre la solución de problemas se aleja de las orientaciones didácticas asumidas por el docente en los planes de estudio para el desarrollo de competencias. De tal manera que contribuyen a la incapacidad para que los estudiantes puedan resolver verdaderos problemas de su entorno, los cuales son muy distintos de los ejercicios que se resuelven de manera mecánica en horas de clase y son propuestos en los talleres.

En otras palabras, la práctica docente se aleja de la propuesta nacional oficial que enmarca desde el constructivismo la prioridad del planteamiento de preguntas y la solución de problemas que permitan al estudiante explorar y construir conocimiento. No cabe duda de que la solución de problemas es una alternativa para el desarrollo de competencias científicas porque parte de la búsqueda de los significados por parte de los estudiantes, mejorando la comprensión de los conceptos y su relación con la realidad. Asimismo, "la resolución de situaciones problemáticas, además de generar actitudes adecuadas hacia las ciencias provoca desarrollos en la independencia cognoscitiva, la capacidad creativa y la construcción de conocimientos en los estudiantes" (García, 2003, p. 39).

Con respecto a las estrategias didácticas, los docentes manifiestan el uso de lecturas, cuadros sinópticos, mapas conceptuales, exposiciones grupales y laboratorios. Desde la perspectiva de los estudiantes se visibiliza el uso del dictado, talleres y trabajos en grupo con más del $50 \%$ en las opciones "siempre" y "casi siempre". El grupo focal realizado con los estudiantes da cuenta de que en el aula de clases predomina la transmisión del conocimiento a partir de la exposición de temas y la aclaración de dudas y los ejemplos. De esta manera, se identifica la tendencia a una educación tradicional donde prima la repetición, la memoria, la exposición oral y está enfocada a la enseñanza y no al aprendizaje. 
Frente a los recursos utilizados sobresale, según los docentes, el uso del tablero, texto guía y fotocopias. Igualmente para los estudiantes, con porcentajes promedio del 70 y $80 \%$. Se identifica el escaso o nulo uso de herramientas tecnológicas como películas, computador, Internet, diapositivas o acetatos, y enciclopedias digitales. Asimismo, cuando manifiestan "Pues la profesora no le podía faltar el libro", "Lo básico que trae un profesor, el marcador, el borrador, el libro, el bolso que nunca le falta", "Jummm, ninguna", "el cuaderno y el lápiz (todos risas)", "el año pasado nos sacó un video". En este sentido, la orientación de la clase de ciencias naturales corresponde a la transmisión de información en donde predominan la explicación del profesor, la lectura del libro de texto y en ocasiones la utilización de recursos audiovisuales, dando al estudiante un rol como receptor de información. Esta postura es confirmada por un docente que manifiesta: "la estrategia didáctica es si viendo con la verdad es la tradicional", "por muchas estrategias nuevas que hayan ahora digo yo que recurrimos siempre a la parte tradicional no somos profesores del momento tecnológico que se está desarrollando en este momento". De esta manera, el modelo pedagógico propuesto por las instituciones educativas no corresponde a lo que realmente hacen los docentes en el aula de clase.

Los aspectos relacionados con la evaluación del área, confirman que este proceso no es entendido en la práctica como la manera como docente y estudiante toman conciencia del desarrollo del proceso de construcción de conocimientos, valores y desarrollo de habilidades, teniendo en cuenta que los criterios de evaluación siempre según los estudiantes corresponden a revisar del cuaderno y evaluar tareas $(63,6 \%)$, aplicar pruebas escritas $(50,91 \%)$ y evaluar exposiciones y sustentaciones $(57,14 \%)$. Por el contrario, para ellos "nunca" se evalúan experimentos e informes de laboratorio $(68,18 \%)$, lo que permite deducir que prevalecen acciones evaluativas tradicionales y no se están empleando espacios para la investigación. Situación contrapuesta a las concepciones declaradas por los docentes, lo que no permitiría emitir un juicio sobre la apropiación del conocimiento en situaciones reales y evidenciar así el desarrollo de las competencias científicas en los estudiantes, además de no propiciar espacios para el trabajo en equipo que redundaría en una evaluación más formativa que propenda por el desarrollo también de competencias ciudadanas. Sin embargo, para el $100 \%$ de los docentes la evaluación es integral porque involucra aspectos cognitivos y actitudinales mediante la incorporación de procesos de autoevaluación y coevaluación, aspecto confirmado por el $64 \%$ de los estudiantes.

\section{Conclusiones}

Al analizar las dos propuestas nacionales (lineamentos curriculares y estándares de competencias) podemos resaltar que ambas coinciden en la importancia de vincular en los procesos de enseñanza y aprendizaje la resolución de problemas y la experimentación. Sin embargo, las orientaciones nacionales no se visibilizan en la propuesta institucional, a pesar de que los lineamientos curriculares y estándares de competencias deben orientar y dirigir el currículo. 
Los resultados analizados permiten concluir que aunque las propuestas pedagógicas nacionales e institucionales para la enseñanza de las ciencias naturales son asumidas desde orientaciones constructivistas, las prácticas docentes riñen con esta realidad, cuando se evidencia que para el docente la construcción de conocimiento no asume al estudiante como protagonista de este proceso y no parte del aprender haciendo; por el contrario, persiste el modelo tradicional de enseñanza. El docente mantiene aún una concepción de ciencia con fuerte influencia positivista, ello tiene implicaciones didácticas, especialmente el transmisionismo y el enciclopedismo en la enseñanza y el memorismo (reproducción acrítica) en el aprendizaje, evidente en las prácticas evaluativas.

De estos datos se puede establecer que no hay congruencia entre lo que afirman los docentes sobre su interés por desarrollar las competencias científicas en los estudiantes y su quehacer pedagógico, porque no se están generando los espacios ni las orientaciones didácticas para propender por su desarrollo.

\section{Referencias}

Ander, E. (1991). El taller, una alternativa para la renovación pedagógica. Buenos Aires: Editorial Magisterio del rio de la Plata.

Bravo, N. (2003). Ensayo: la didáctica problémica. Recuperado el 10 de julio de 2011 desde http://acreditacion.unillanos.edu. co/contenidos/NESTOR\%20BRAVO/Cuar-
ta\%20sesi\%F3n/Ensayo_pedagogia_problematica.pdf

Cañas, A., Martín, M. y Nieda, J. (2007). Competencia en el conocimiento y la interacción con el mundo físico. La competencia científica. Madrid: Alianza Editorial.

D'amore, B. et ál. (2008). Competencias y matemática. Bogotá: Cooperativa Editorial Magisterio.

Escobedo, H. (2001). Desarrollo de competencias básicas para pensar científicamente. Una propuesta didáctica para las ciencias naturales. Bogotá: Colciencias.

Flores, F., Gallego, L., Bonilla, X., López,. L. y García, B. (2007). Concepciones sobre la naturaleza de la ciencia de los profesores de biología del nivel secundario. Revista Mexicana de la Investigación Educativa, 32 (12). Recuperado el 23 de julio de 2011 desde http://redalyc.uaemex.mx/ pdf/140/14003217.pdf

García, J. (2003). Didáctica de las ciencias resolución de problemas y desarrollo de la creatividad. Bogotá: Cooperativa Editorial Magisterio.

Hernández, C. (2005). ¿Qué son las competencias cientificas?. Foro Educativo Nacional. Competencias Científicas. Recuperado el 
4 de diciembre de 2009 desde http://www. colombiaaprende.edu.co/html/mediateca/1607/article-128237.html

Maldonado G., M. (2002). Las competencias: una opción de vida. Bogotá: ECOE Ediciones.

Ministerio de Educación Nacional (MEN). (2004). Estándares básicos de competencias en ciencias naturales y en ciencias sociales. Recuperado el 15 de agosto de 2009 desde http://www.mineducacion.gov. co/cvn/1665/articles-116042_archivo_pdf3. pdf

Ministerio de Educación Nacional (MEN). (1998). Lineamientos curriculares. Ciencias naturales y educación ambiental. Bogotá: Cooperativa Editorial Magisterio.

Mora, W. (1997). Naturaleza del conocimiento científico e implicaciones didácticas. Revista Educación y Pedagogía, 18(IX). MedeIlín: Facultad de Educación - Universidad de Antioquia.

Porlán, R. (1995). Constructivismo y escuela. Hacia un modelo de enseñanza-aprendizaje basado en la investigación. Sevilla: Diada Editora.
Quintanilla, M. (2005). Competencias centíficas. Identificación y caracterización de competencias científicas en el aula, ¿qué cambia en la enseñanza y en los nuevos modelos de conocimiento? Foro Educativo Nacional - Ministerio de Educación Nacional. Recuperado el 4 de diciembre de 2009 desde http://www.colombiaaprende.edu.co/ html/mediateca/1607/articles-128237_archivo.pdf

República de Colombia. Ley General de Educación. Ley 115 de 1994.

Soto, C. (2002). Metacognición, cambio conceptual y enseñanza de las ciencias. Bogotá: Cooperativa Editorial Magisterio.

Tobón. S. (2006). Formación basada en competencias. Pensamiento complejo, diseño curricular y didáctica. Bogotá: Ecoe Ediciones.

Zambrano, L. A. (2005). Didáctica, pedagogía y saber. Bogotá: Editorial Magisterio. 
Anexo 1. Principales hallazgos en la documentación de la propuesta nacional oficial e institucional

\begin{tabular}{|c|c|c|c|}
\hline Fuente & Concepción competencia & $\begin{array}{l}\text { Competencias } \\
\text { básicas y } \\
\text { científicas }\end{array}$ & $\begin{array}{c}\text { Enfoque } \\
\text { pedagógico }\end{array}$ \\
\hline $\begin{array}{l}\text { Lineamientos } \\
\text { curriculares } \\
\text { (MEN, 1998) }\end{array}$ & $\begin{array}{l}\text { - Desarrollar las competencias necesarias } \\
\text { para identificar, anticipar y resolver } \\
\text { - Capacidades, aptitudes, saber conocer, } \\
\text { saber hacer, saber ser } \\
\text { - Formación científica básica, formación } \\
\text { para el trabajo, formación ética }\end{array}$ & $\begin{array}{l}\text { Interpretar, } \\
\text { argumentar } \\
\text { proponer, indagar } \\
\text { explicar, } \\
\text { comunicar }\end{array}$ & Constructivismo \\
\hline $\begin{array}{l}\text { Estándares de } \\
\text { competencias } \\
\text { (MEN, 2004) }\end{array}$ & $\begin{array}{l}\text { - Conocimientos, habilidades, } \\
\text { destrezas, comprensiones, actitudes y } \\
\text { disposiciones. Saber, saber hacer } \\
\text { - Me aproximo al conocimiento como } \\
\text { científico natural } \\
\text { - Manejo de conocimientos propios de } \\
\text { las ciencias naturales } \\
\text { - Desarrollo de compromisos personales } \\
\text { y sociales }\end{array}$ & $\begin{array}{l}\text { Interpretar, } \\
\text { argumentar } \\
\text { proponer, } \\
\text { indagar explicar, } \\
\text { comunicar }\end{array}$ & Constructivismo \\
\hline PEI & $\begin{array}{l}\text { El término "competencia" aparece en } \\
\text { varios componentes del PEI pero no } \\
\text { existe una coherencia en el desarrollo del } \\
\text { documento }\end{array}$ & & $\begin{array}{l}\text { Constructivismo } \\
\text { Humanismo }\end{array}$ \\
\hline $\begin{array}{l}\text { Plan de } \\
\text { estudios }\end{array}$ & Conocimientos, desempeños, ser & $\begin{array}{l}\text { Interpretar, } \\
\text { argumentar } \\
\text { proponer, } \\
\text { indagar explicar, } \\
\text { comunicar }\end{array}$ & Constructivismo \\
\hline Docentes & Saber saber, saber hacer, saber ser & $\begin{array}{l}\text { Interpretar, } \\
\text { argumentar } \\
\text { proponer, } \\
\text { indagar explicar, } \\
\text { comunicar } \\
\text { experimentar }\end{array}$ & Tradicional \\
\hline
\end{tabular}


Anexo 2. Algunas percepciones de docentes y estudiantes sobre la práctica de aula

\begin{tabular}{|c|c|c|}
\hline Didáctica & Docente & Estudiante \\
\hline $\begin{array}{l}\text { Laboratorios y } \\
\text { experimentación }\end{array}$ & $\begin{array}{l}\text { "entonces en el laboratorio el } \\
\text { estudiante va a experimentar } \\
\text { también que esa es la forma } \\
\text { de ir logrando la competencia } \\
\text { científica" } \\
\text { "los contenidos básicos que se } \\
\text { desarrollan en el área como tal se } \\
\text { llevan a la parte práctica pues la } \\
\text { parte práctica es experimentar y } \\
\text { comprobar la teoría" } \\
\text { "entonces se hace una práctica de } \\
\text { laboratorio relacionada con ese } \\
\text { tema" }\end{array}$ & $\begin{array}{l}\text { "ningún experimento nosotros no hicimos } \\
\text { ningún experimento" } \\
\text { "Solamente lo único que nos hacían venir al } \\
\text { laboratorio era a las evaluaciones" } \\
\text { "nunca hicimos una práctica de laboratorio } \\
\text { con el profesor, ni experimentos" } \\
\text { "porque la idea de nosotros era que de } \\
\text { pronto en octavo pues decían que octavo } \\
\text { ya eran puros experimentos y experimentos } \\
\text { pues si nosotros queríamos experimentar } \\
\text { eso y no la oportunidad no se nos dio" }\end{array}$ \\
\hline $\begin{array}{l}\text { Talleres y trabajos } \\
\text { en grupo }\end{array}$ & $\begin{array}{l}\text { "se le aplica un taller que se } \\
\text { trabaja en la misma clase" } \\
\text { "mucho apoyo, si, talleres" } \\
\text { "después se hace el taller" }\end{array}$ & $\begin{array}{l}\text { "siempre era talleres copiar del libro } \\
\text { evaluación y sale", "y los talleres los que } \\
\text { estaban en los libros" } \\
\text { "así siempre que dejaba un taller dejaba que } \\
\text { se armara por ejemplo un grupo de cuatro o } \\
\text { tres estudiantes a realizar el taller en clase y } \\
\text { pues a la clase siguiente ya calificaba" } \\
\text { "nos reunía en grupos de siete y como las } \\
\text { cartillas no son muchas nos reunía en grupos } \\
\text { de a siete o de a fila y le entregaba un libro, } \\
\text { a cada grupo le entregaba un tema" }\end{array}$ \\
\hline
\end{tabular}




\begin{tabular}{|c|c|c|}
\hline Didáctica & Docente & Estudiante \\
\hline $\begin{array}{l}\text { Planteamiento } \\
\text { y solución de } \\
\text { problemas }\end{array}$ & $\begin{array}{l}\text { "también hay veces que hago } \\
\text { preguntas que las deben saber y } \\
\text { ellos se quedan como en el aire, } \\
\text { entonces le digo por ejemplo } 5 \\
\text { al que de la respuesta y eso los } \\
\text { incentiva también a ellos" } \\
\text { "y retomamos lo que resumiendo } \\
\text { a través de preguntas" } \\
\text { "a veces retomo la clase anterior } \\
\text { donde los muchachos participan, } \\
\text { genero preguntas sobre ¿qué } \\
\text { recuerdan?" } \\
\text { "se hace las preguntas las dudas } \\
\text { que el estudiante tenga" } \\
\text { "con problemas simulados } \\
\text { situaciones simuladas" }\end{array}$ & $\begin{array}{l}\text { "el profesor repetía e preguntaba sobre el } \\
\text { tema anterior de la clase pasada" } \\
\text { "que preguntara que son la neuronas } \\
\text { al iniciar el tema para que el estudiante } \\
\text { digamos si tuviera unas nociones, no, no } \\
\text { preguntaba nada de eso después de que } \\
\text { enseñaba el tema era que ella hacía eso" } \\
\text { "Y son los problemas y las preguntas del } \\
\text { libro" }\end{array}$ \\
\hline Investigación & $\begin{array}{l}\text { "trabajar mucho la forma de que } \\
\text { ellos lean que investiguen" } \\
\text { "genero proyectos" }\end{array}$ & $\begin{array}{l}\text { "Yo no me acuerdo haber hecho eso" } \\
\text { "Nosotros nunca planteamos proyectos de } \\
\text { investigación y tampoco no nos surgió la } \\
\text { idea de plantear temas con tan poco interés" }\end{array}$ \\
\hline
\end{tabular}

